\title{
The Detection, Symbol Period and Chip Width Estimation of DSSS Signals Based on Delay-Multiply, Correlation and Spectrum Analysis
}

\author{
Zhanqi DONG and Hanying HU
}

\begin{abstract}
Based on the prominent characteristic that the delay-multiply signals of the direct sequence spread spectral (DSSS) signals are periodical signals, the paper proposes an efficient method for the detection of DSSS signals: the detection, symbol period and chip width estimation of DSSS signals based on delay-multiply, correlation and spectrum analysis. The computer simulations show that the method can not only fit the need of the low signal to noise ratio which is quite common in the detection of DSSS signals, but also can give the estimations of the symbol period and chip width in a simple way. Especially, the proposed method can give an exact estimation of the symbol period by increasing the processed sampling data when the sampling rate is fixed, which is very important for the detection of DSSS signals.
\end{abstract}

Index Terms-Correlation and Spectrum Analysis, DelayMultiply, DSSS signals, Enhancing Detection Based on FFT, Symbol Period and Chip Width.

\section{INTRODUCTION}

DSSS communication is widely used in military communication and modern commercial communication for its good traits, such as noise-like, low power density spectrum and low probability of intercept. Since the application of DSSS communication the detection of the signals has been an important content for the detection of communication signals. In order to achieve the detection of DSSS signals in a good way, the paper, after researching on the periodicity of the delay-multiply signal of DSSS signals detailedly, proposes a good method for the detection of the DSSS signals: the detection of DSSS signals based on delay-multiply, correlation and spectrum analysis.

Manuscript received November 9, 2006.

Zhanqi DONG is with the Zhengzhou Information Science and Engineering Institute, Zhengzhou, 450002, China (phone: 86-371-63530541; fax: 86-37163530551; e-mail: llmmxxgc@126.com), he is also with Nanyang Institute of Technology, Nanyang, 473004, China (Address: No.80, Changjiang Road, Nanyang, China )

Hanying $\mathrm{HU}$ is with the Zhengzhou Information Science and Engineering Institute, Zhengzhou, 450002, China (phone: 86-371-63531332; e-mail: huhanying@vip.sina.com).

\section{The PREPRocessing BASED ON DELAY-Multiply}

Generally, the baseband form of the DSSS signals may be written as:

$$
s(t)=d(t) c(t)=\sum_{j=-\infty}^{\infty} d_{j} \sum_{k=0}^{N-1} c_{k} g\left(t-j T_{s}-k T_{c}\right)
$$

Where $d_{j}, c_{k} \in\{1,-1\}, d_{j}$ is the date sequence, $T_{s}$ is the period of a date symbol, $c_{k}(k=0,1, \cdots, N-1)$ is the $k$-th chip of the spread spectral pseudo-random sequence, $T_{c}$ is the duration of the chip, $T_{s}=N T_{c}, N$ is the length of the spread spectral pseudo-random sequence, $g(t)=\operatorname{rect}\left(t / T_{c}\right)$ is the chip waveform.

In formula (1), $d(t)$ is random, $c(t)$ is pseudo-random/ periodical, if we do $T_{c}$-delay-multiply on $s(t)$ shown in (1), there is

$$
u_{s}(t)=d(t) d\left(t-T_{c}\right) c(t) c\left(t-T_{c}\right)
$$

For formula (2), because there are $d(t)=d\left(t-T_{c}\right)$ for any data symbol except for the first chip, $u_{s}(t)$ may be looked as the baseband signal corresponding to the periodical repetition copy of the relative transform sequence of the spread spectral pseudo-random sequence, in which the value-taking of $u_{s}(t)$ is identical for all of the data symbols expect for the first chip.

Even more, if we note the relative transform sequence of the spread spectral pseudo-random sequence as:

$$
\vec{C}_{0}=\left[x_{0}, X_{1}, \cdots, x_{N-1}\right]
$$

Where $x_{0}=C_{0}, x_{k}=C_{k} \cdot C_{k-1}, 1 \leq k \leq N-1$.

Then the relative transform sequence corresponding to the data symbol $d_{j}$ will be:

$$
\vec{C}_{u_{0}}=\left[\left(d_{j-1} \cdot c_{N-1}\right) \cdot\left(d_{j} \cdot c_{0}\right), x_{1}, \cdots, x_{N-1}\right]
$$

As such, $u_{s}(t)$ shown in formula (2) may be looked as the signal which is generated by the repeating of $\vec{C}_{u_{0}}$. For such a periodical signal, for $T_{s}>>T_{c}$ if we ignore the difference at 
the beginning of every data symbol, then $u_{s}(t)$ will be a periodical signal, whose period is $T_{s}$.

In the above analyzing, we assume $T_{c}$ is known. In actual detection of DSSS signals there is commonly no prior knowledge of $T_{c}$, and such, if we do delay-multiply $\left(0<t_{d}\right)$ on $s(t)$ shown in formula (1), the results will be as follows:

$$
\begin{aligned}
& \text { For } 0<t_{d}<T_{c}, \\
& u_{s}^{\prime}(t)=\left\{\begin{aligned}
d(t) d\left(t-t_{d}\right) c(t) c\left(t-t_{d}\right), \\
\quad(j N+k) T_{c}<t \leq(j N+k) T_{c}+t_{d} \\
1, \quad(j N+k) T_{c}+t_{d}<t \leq(j N+k) T_{c}+T_{c}
\end{aligned}\right.
\end{aligned}
$$

The sequence corresponding to the signal $u_{s}^{\prime}(t)$ shown in formula (5) is the interleaving sequence of the relative transform sequence shown in formula (4) and an all 1 sequence. It is also a periodical signal except that there exists random when $t$ falls into $\left[j N \cdot T_{c}, j N \cdot T_{c}+t_{d}\right)$.

$$
\begin{aligned}
& \text { For } T_{c}<t_{d} \leq 2 T_{c}, \\
& u_{s}^{\prime}(t)=d(t) d\left(t-t_{d}\right) c(t) c\left(t-t_{d}\right) \\
& =\left\{\begin{array}{c}
d_{j} \cdot d_{j-1} \cdot c_{k} \cdot c_{\bmod (N, k-2)}, \\
\quad(j N+k) T_{c}<t<(j N+k-1) T_{c}+t_{d}, k \leq 1 \\
d_{j} \cdot d_{j-1} \cdot c_{0} \cdot c_{N-1}, \\
\quad(j N-1) T_{c}+t_{d}<t<(j N+1) T_{c}, k=0 \\
c_{1} \cdot c_{0}, \\
j N T_{c}+t_{d}<t<(j N+2) T_{c}, k=1 \\
c_{k} \cdot c_{k-2}, \\
\quad(j N+k) T_{c}<t<(j N+k-1) T_{c}+t_{d}, k \geq 2 \\
c_{k} \cdot c_{k-1}, \\
(j N+k-1) T_{c}+t_{d}<t<(j N+k+1) T_{c}, k \geq 2
\end{array}\right.
\end{aligned}
$$

In a certain sense, the signal $u_{s}^{\prime}(t)$ shown in formula (6) is the baseband signal corresponding to an another relative transform sequence of the spread spectral pseudo-random sequence which is like the relative transform sequence shown in formula (4) in some way. Although the signal is more complex than the signal shown in formula (2), it is a quasi-periodicity signal for that there shows randomicity only in the first chip and part of the second chip for every data symbol. Especially, when $t_{d}=2 T_{c}$, there shows randomicity in the first-two-chip for every data symbol, and the corresponding relative transform sequence is just $\vec{C}_{u_{0}^{\prime}}=\left[\left(d_{j-1} \cdot c_{N-2}\right) \cdot\left(d_{j} \cdot c_{0}\right),\left(d_{j-1} \cdot c_{N-1}\right) \cdot\left(d_{j} \cdot c_{1}\right)\right.$, $\left.x_{2}^{\prime}, \cdots, x_{N-1}^{\prime}\right], x_{k}^{\prime}=c_{k} \cdot c_{k-2}, k \geq 2$.

For $2 T_{c}<t_{d} \leq(N-1) T_{c}$, although the delay-multiply signal is much more complex than the delay-multiply signal shown in formula (2), formula (5) or formula (6), and the periodicity is weaken with the increase of the time-delay $t_{d}$ $\left(2 T_{c}<t_{d} \leq(N-1) T_{c}\right)$, the delay-multiply signal has periodicity to a certain extent and the characteristic of the signal structure is similar to the signal shown in formula (6).
Especially, in some sense, the delay-multiply signal of the signal shown in formula (1) is absolutely random or just alike the original DSSS signal only when $(N-1) T_{c}<t_{d}$. Thus, in theory, we can get periodical signal by doing delay-multiply operation on DSSS signal ( $t_{d}$ is less than $T_{s}=N T_{c}$ ).

\section{The Autocorrelation OF the Delay-Multiply SIGNAL}

For the delay-multiply signal of the DSSS signals possesses classical periodicity, and by doing autocorrelation we can reveal the character of a periodical signal ${ }^{[2,3]}$, we discuss the autocorrelation of the delay-multiply signal of the DSSS signals in this section. Especially, for the sake of simpleness, here we only do some analysis on the autocorrelation of the delay-multiply signal of the DSSS signals with several distinct $t_{d}$.

First, when $t_{d}=T_{c}$, based on the discussion of section II, we know the delay-multiply signal of the DSSS signals is the signal corresponding to the periodical repetition of the relative transform sequence $\vec{C}_{u_{0}}$, and the autocorrelation may be expressed as follows ${ }^{[1,2]}$ :

$$
R(\tau) \approx\left\{\begin{array}{l}
1-\frac{N+n}{N T_{c}}|\tau|, 0 \leq|\tau| \leq T_{c} \\
\left(1-\frac{1+n /(N-1)}{T_{c}}|| \tau\left|-j N T_{c}\right|\right) \cdot \frac{N-1}{N}, \\
0 \leq \| \tau\left|-j N T_{c}\right| \leq T_{c}, j=1,2, \cdots \\
-\frac{n}{N}, \quad \text { other } \tau
\end{array}\right.
$$

Where $n$ is the sum difference between the "- $1 / 0$ " elements and the " 1 " elements in the relative transform sequence $\vec{C}_{u_{0}}$, commonly, $n<<N$.

From formula (7), it is clear that the autocorrelation of the delay-multiply signal is a periodical function and it will show obvious peak when the autocorrelation time delay parameter $\tau$ is equal to $N T_{c}$ (or $T_{s}$ ) or its times when $t_{d}=T_{c}$.

Next, when $0<t_{d}<T_{c} / 2$, from section II, we know the delay-multiply signal of the DSSS signals is the signal corresponding to the periodical repetition of the interleaving sequence of the relative transform sequence shown in formula (4) and an all 1 sequence, and especially, the duration of any element of $\vec{C}_{u_{0}}$ is $t_{d}$, the duration of any " 1 " elements of the all 1 sequence is $T_{c}-t_{d}$. And the autocorrelation of the delay-multiply signal may be written as: 


$$
R(\tau) \approx\left\{\begin{array}{c}
1-\frac{(N-n) \cdot 2 t_{d} / T_{c}+n}{N t_{d}}|\tau|, 0 \leq|\tau| \leq t_{d} \\
\left(1-\frac{1+\left(2 t_{d} / T_{c}-1\right) \cdot(N-n) /(N-1)}{t_{d}}|| \tau\left|-j N T_{c}\right|\right) \\
\cdot \frac{N-1}{N}, \quad 0 \leq|| \tau\left|-j N T_{c}\right| \leq t_{d}, j=1,2, \cdots \\
\left(1-\frac{(N-n) \cdot t_{d} /\left(T_{c}-t_{d}\right)+n}{N t_{d}}|| \tau\left|-j N T_{c}-k T_{c}\right|\right) \\
\cdot \frac{T_{c}-t_{d}}{T_{c}} \cdot \frac{N-1}{N}, \quad 0 \leq|| \tau\left|-j N T_{c}-k T_{c}\right| \leq t_{d}, \\
j=0,1, \cdots, k=1, \cdots, N-1 \\
\left(1-2 t_{d} / T_{c}\right) \cdot(N-n) / N, \\
t_{d}<|| \tau\left|-j N T_{c}-k T_{c}\right| \leq T_{c}-t_{d}
\end{array}\right.
$$

Third, when $T_{c} / 2 \leq t_{d}<T_{c}$, the autocorrelation of the delay-multiply signal shown in formula (5) may be written as:

$$
R(\tau) \approx\left\{\begin{array}{l}
1-\frac{N+n\left(2 t_{d}-T_{c}\right) / T_{c}}{N t_{d}}|\tau|, 0 \leq|\tau| \leq t_{d} \\
\left(1-\frac{1+n\left(2 t_{d}-T_{c}\right) /\left(T_{c} \cdot(N-1)\right)}{t_{d}}|| \tau\left|-j N T_{c}\right|\right) \\
\cdot \frac{N-1}{N}, \quad 0 \leq|| \tau\left|-j N T_{c}\right| \leq t_{d}, j=1,2, \cdots \\
\left(1-\frac{N+n\left(2 t_{d}-T_{c}\right) /\left(T_{c}-t_{d}\right)}{N t_{d}}|| \tau\left|-j N T_{c}-k T_{c}\right|\right) \\
\cdot \frac{T_{c}-t_{d}}{T_{c}} \cdot \frac{N-1}{N}, 0 \leq|| \tau\left|-j N T_{c}-k T_{c}\right| \leq T_{c}-t_{d}, \\
\frac{n}{N} \cdot\left(T_{c}-2 t_{d}\right) / T_{c}, \\
t_{d}<\| \tau\left|-j N T_{c}-k T_{c}\right| \leq T_{c}-t_{d}, k=2, \cdots, N-2
\end{array}\right.
$$

From formula (8) and (9), it is clear that the autocorrelation of the delay-multiply signal of the DSSS signals will also show obvious peaks when the autocorrelation time delay parameter $\tau$ is equal to $N T_{c}$ (or $T_{s}$ ) or its times when $0<t_{d}<T_{c}$.

In addition, when $T_{c}<t_{d}<2 T_{c}$, based on the discussion of section II, we know the delay-multiply signal of the DSSS signals is the signal corresponding to the periodical repetition of the interleaving sequence of $\vec{C}_{u_{0}^{\prime}}$ and $\vec{C}_{u_{0}}$. The autocorrelation of the delay-multiply signal will take peak-value when the autocorrelation time delay parameter $\tau$ is equal to $N T_{c}$ (or $T_{s}$ ) or its times. In particular, when $t_{d}=2 T_{c}$, the delay-multiply signal of the DSSS signals is the signal corresponding to the periodical repetition of the relative transform sequence of $\vec{C}_{u_{0}^{\prime}}$, and the autocorrelation may be written as:

$$
R(\tau)=\left\{\begin{array}{l}
1-\frac{N+n^{\prime}}{N T_{c}}|\tau|, 0 \leq|\tau| \leq T_{c} \\
\left(1-\frac{1+n^{\prime} /(N-2)}{T_{c}}|| \tau|-| j\left|N T_{c}\right|\right) \cdot \frac{N-2}{N}, \\
\quad 0 \leq|| \tau|-| j\left|N T_{c}\right| \leq T_{c}, j \neq 0 \\
-\frac{n^{\prime}}{N}, \quad \text { other } \tau
\end{array}\right.
$$

Where $n^{\prime}$ is the sum difference between the "-1/0" elements and the " 1 " elements in the relative transform sequence $\vec{C}_{u_{0}^{\prime}}$, in general, $n^{\prime}<<N$.

With formula (10), it is clear that, when $t_{d}=2 T_{c}$, the autocorrelation of the delay-multiply signal is also a periodical function and it will show obvious peak when the autocorrelation time delay parameter $\tau$ is equal to $N T_{c}$ (or $T_{s}$ ) or its times.

All in all, the autocorrelation of the delay-multiply signal of the DSSS signals is a kind of periodical function and it will take peak value when the autocorrelation time delay parameter $\tau$ is equal to $N T_{c}$ (or $T_{s}$ ) or its times. In the detection of the DSSS signals, we may ponder to achieve the detection by the means of delay- multiply and correlation analysis.

\section{The Characteristic OF THE DELAY-Multiply Signal OF DSSS SignAL IN NOISE CONTEXT}

In the above analysis we show the theory base to detect DSSS signal by delay-multiply and correlation analysis when noise is absent. In actual detection of DSSS signal, the signal is polluted by noise, so to detect DSSS signal by delay-multiply and correlation analysis we need to analyze the characteristic of the delay-multiply signal of the noise-polluted DSSS signal.

Commonly, the noise-polluted DSSS signal may be expressed as:

$$
\begin{aligned}
r(t) & =d(t) c(t)+n(t) \\
& =\sum_{j=-\infty}^{\infty} d_{j} \sum_{k=0}^{N-1} c_{k} g\left(t-j T_{s}-k T_{c}\right)+n(t)
\end{aligned}
$$

Where $n(t)$ is zero mean noise which is irrelevant to $d_{j}$ and $C_{k}$.

Do delay-multiply operation (Commonly, $t_{d}<<T_{s}$ ) on the signal shown in formula (11), we have 


$$
\begin{aligned}
u(t)= & d(t) d\left(t-t_{d}\right) c(t) c\left(t-t_{d}\right) \\
& +n(t) d\left(t-t_{d}\right) c\left(t-t_{d}\right) \\
& +n\left(t-t_{d}\right) d(t) c(t)+n(t) n\left(t-t_{d}\right) \\
& =d(t) d\left(t-t_{d}\right) c(t) c\left(t-t_{d}\right)+r_{n}^{\prime}(t) \\
& =u_{s}^{\prime}(t)+r_{n}^{\prime}(t)
\end{aligned}
$$

Where $r_{n}^{\prime}(t)=n(t) d\left(t-t_{d}\right) c\left(t-t_{d}\right)+n\left(t-t_{d}\right) d(t)$ $c(t)+n(t) n\left(t-t_{d}\right)$.

For formula (12), first, $u_{s}^{\prime}(t)$ is a periodical signal (quasi-periodicity), second, $d_{j}$ and $c_{k}$ are irrelevant to $n(t)$, and $n(t)$ is irrelevant to $n\left(t-t_{d}\right)$ when the bandwidth of the non-cooperative receiver front end is no less than $1 / t_{d}$, so $r_{n}^{\prime}(t)$ is a zero mean band-limited white gaussian noise process, it is irrelevant to $u_{s}^{\prime}(t)$. Such, in some sense, $u(t)$ is still a periodical signal, and we can find its characteristic by correlation analysis.

\section{Computer Simulation}

To test the feasibility of the theory analysis, here do some simulations on a DSSS communication signal whose spread spectrum pseudo random sequence is "- $1,-1,-1,-1,1,-1,1,-1,1$, $1,1,-1,1,1,-1,-1,-1,1,1,1,1,1,-1,-1,1,1,-1,1,-1,-1,1$ " which is derived from the original polynomial $p(x)=x^{5}+x+1$. In particular, when the signal to noise ratio is $-10 \mathrm{~dB}$, the simulation results under the environment of Matlab are shown in Fig.1 (Fig.1.a, Fig.1.b and Fig.1.c are corresponding to the results of $t_{d}=T_{c} / 2, t_{d}=3 T_{c} / 4$, and $t_{d}=T_{c}$ respectively).
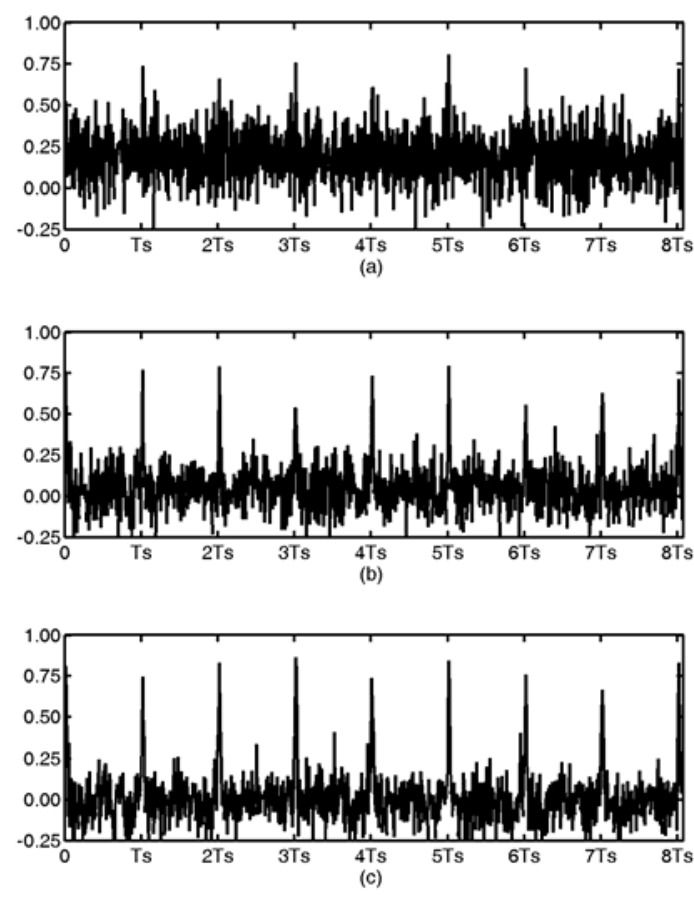

Figure 1. The autocorrelation of the delay-multiply signal of the DSSS signal
With Fig.1 it is clear that the autocorrelation of the delaymultiply signal of the DSSS signal does show obvious correlation peaks when the time delay parameter $\tau$ is equal to the symbol period or its times, and the space of the correlation peaks is equal to the symbol period $T_{s}$. So, in the detection of DSSS signal, we can achieve the detection by examining the presence of the correlation peaks, and especially, we can estimate the period of the data symbol at the same time by examining the space of the correlation peaks.

Especially, by contrasting the three sub-figures in Fig.1, we may find that the value-taking of the time-delay $t_{d}$ has important effect on the performance of the detection. With the increase of $t_{d}, T_{c} / 2<t_{d}<T_{c}$, the performance improves quickly, and the centre of the value-taking of the autocorrelation shifts down gradually (except for $\tau$ is equal to $i T_{s}, i$ is an integer), in the end it stops at a certain position under " 0 " line.

By the way, we also do some simulations on the DSSS signal with other $t_{d}\left(0<t_{d}<T_{c} / 2\right.$ or $\left.T_{c}<t_{d}<N T_{c}=T_{s}\right)$, the results show that we can achieve the detection of DSSS signal by delay-multiply and correlation analysis in a certain degree while $T_{c} / 3<t_{d}<T_{c} / 2$ or $T_{c}<t_{d} \leq T_{s} / 2$, nevertheless the optimal performance is gotten when $t_{d}$ is close to $T_{c}$.

\section{The ENHANCING Detection of DSSS SignaLS AND THE ESTIMATION OF THE SYMBol PERIOD AND CHIP WIDTH}

According to the analysis of part $\mathrm{V}$ we know that the detection of DSSS signal by delay-multiply and correlation analysis do can achieve the detection of DSSS signal, but the good performance is gotten only when the value-taking of the time-delay $t_{d}$ is close to $T_{c}$. In actual detection, for there is usually no prior knowledge of $T_{c}$, and especially bases on the fact that spectrum analysis can reveal the character of a periodical signal in a good way, we may ponder to improve the performance of the detection by doing FFT analysis on the autocorrelation of the delay-multiply signal. Fig.2 displays the results of the FFT of the autocorrelation shown in Fig.1 (In Fig.2, $F_{e}$ is the sampling rate, $F_{c}=1 / T_{c}$ and it is the chip rate, $F_{s}=1 / T_{s}$, it is the data symbol rate. Fig.2.a, Fig.2.b and Fig.2.c are corresponding to the results of $t_{d}=T_{c} / 2$, $t_{d}=3 T_{c} / 4$, and $t_{d}=T_{c}$ respectively). 

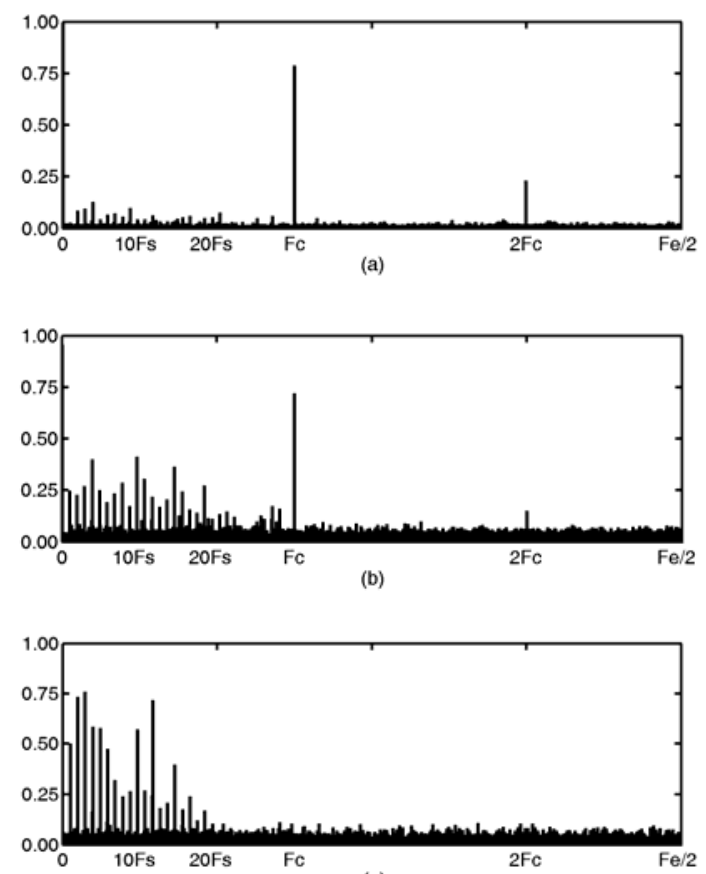

(c)

Figure 2. The FFT of the autocorrelation of the delay-multiply signal of the DSSS signal

With Fig.2 we can find that: A. By performing delay-multiply, correlation and FFT spectrum analysis on DSSS signal we can get discrete spectrum lines, and importantly, the discrete spectrum lines lies in the multiples of the data symbol rate and the chip rate. In the detection of DSSS signal we can achieve the detection by examining the existence of the discrete spectrum lines, and especially, we can achieve the estimation of the symbol period and the chip width by examining the space of the discrete spectrum lines at the same time. B. Just like the detection based on delay-multiply and correlation analysis, the value-taking of the time-delay $t_{d}$ has important effect on the performance of the detection which is based on delay-multiply, correlation and FFT spectrum analysis, nevertheless there exists difference: Although the detection performance aiming at data symbol period degrades with the decrease of $t_{d}$ when $0<t_{d} \leq T_{c}$, we can get obvious discrete spectrum lines corresponding to the chip rate in a quite large range of $0<t_{d} \leq T_{c}$. So we can still achieve the detection of DSSS signal by delay-multiply, correlation and FFT spectrum analysis when $t_{d}$ is much less than $T_{c}$. Especially, by contrasting Fig. 2 with Fig. 1 we can find that, after the operation of FFT, the characteristic of DSSS signal is enhanced, the unexpected noise is restrained, and the information of the chip width is revealed, and very importantly, all of these make the detection of DSSS signal and the parameter estimation more easily.

By the way, and quite importantly, by using the detection method proposed above, we can get the exact estimation of the symbol period by increasing the processed sampling data while the sampling rate is fixed.

\section{CONCLUSION}

The blind detection method proposed in this paper makes full use of the characteristic of DSSS signal, it has high robustness for the detection of DSSS signal in noise context, by using it we can achieve the detection of DSSS signal and the estimation of the key parameters (symbol period and chip width), and its application is significant for the detection of DSSS signal.

\section{REFERENCES}

[1] A. J. Viterbi, "CDMA: Principles of spread spectrum communication," New York: Addison-WeSley, 1995.

[2] LIN Kexiang, WANG Yifei. "The Principle \& Application of the Pseudo-random Sequence (section 4.4)". PTPress: Posts and Telecommunications Press (Beijing, China), 1978.

[3] The institute of information physics, Nanjing University. "The Detection Technology of Weak Signals (section 7.2.H)”. The Higher Education Press, Beijing, China, 1991.

[4] MENG Jian and HU Laizhao, "The Correlation Cumulation Technology of the DSSS Singals,” Electronic Warfare (China). VOL.16 NO.2, pp.1 5, 2001.

[5] [5] JOHN F. KUEHLS, EVAGGELOS GERANIOTS, "Presence Detection of Binary-Phase-Shift-Keyed and Direct-Sequence Spread-Spectrum Signals Using a Prefilter-Delay-and-Multiply Device,” IEEE Journal on Selected Areas in Communications, VOL.8 NO.5, pp.915-933, June 1990. 